\title{
BMJ Open Effect of a low-salt diet on chronic kidney disease outcomes: a systematic review and meta-analysis
}

\author{
Honghong Shi (D) , ${ }^{1}$ Xiaole Su, ${ }^{1}$ Chunfang Li, ${ }^{2}$ Wenjuan Guo, ${ }^{1}$ Lihua Wang ${ }^{1}$
}

To cite: Shi H, Su X, Li C, et al. Effect of a low-salt diet on chronic kidney disease outcomes: a systematic review and meta-analysis. BMJ Open 2022;12:e050843. doi:10.1136/ bmjopen-2021-050843

- Prepublication history and additional supplemental material for this paper are available online. To view these files, please visit the journal online (http://dx.doi.org/10.1136/ bmjopen-2021-050843)

Received 05 March 2021 Accepted 24 November 2021

Check for updates

(c) Author(s) (or their employer(s)) 2022. Re-use permitted under CC BY-NC. No commercial re-use. See rights and permissions. Published by BMJ.

${ }^{1}$ Renal Division, Shanxi Medical University Second Affiliated Hospital, Taiyuan, Shanxi, China

${ }^{2}$ Renal Divison, Shanxi

Cardiovascular Hospital, Taiyuan, Shanxi, China

Correspondence to

Dr Lihua Wang;

lihuawang236@126.com

\section{ABSTRACT}

Objective The benefits of a low-salt diet for patients with chronic kidney disease (CKD) are controversial. We conducted a systematic review and meta-analysis of the effect of a low-salt diet on major clinical outcomes.

Design Systematic review and meta-analysis.

Data sources MEDLINE by Ovid, EMBASE and the Cochrane Library databases.

Eligibility criteria for selecting studies We included randomised controlled trials (RCTs) and cohort studies that assessed the effect of a low-salt diet on the renal composite outcomes (more than $50 \%$ decline in estimated glomerular filtration rate (eGFR) during follow-up, doubling of serum creatinine or end-stage renal disease), rate of eGFR decline, change in proteinuria, all-cause mortality events, cardiovascular (CV) events, and changes in systolic blood pressure and diastolic blood pressure.

Data extraction and synthesis Two independent researchers extracted data and evaluated their quality. Relative risks (RRs) with 95\% Cls were used for dichotomous data. Differences in means (MDs) or standardised mean differences (SMDs) with 95\% Cls were used to pool continuous data. We used the Cochrane Collaboration risk-of-bias tool to evaluate the quality of RCTs, and Newcastle-0ttawa Scale to evaluate the quality of cohort studies.

Results We found 9948 potential research records. After removing duplicates, we reviewed the titles and abstracts, and screened the full text of 230 publications. Thirty-three studies with 101077 participants were included. A lowsalt diet produced a $28 \%$ reduction in renal composite outcome events (RR: $0.72 ; 95 \% \mathrm{Cl}$ : 0.58 to 0.89 ). No significant effects were found in terms of changes in proteinuria (SMD: $-0.71 ; 95 \% \mathrm{Cl}:-1.66$ to 0.24 ), rate of eGFR (decline MD: $1.16 ; 95 \% \mathrm{Cl}:-2.02$ to 4.33 ), risk of all-cause mortality (RR: $0.92 ; 95 \% \mathrm{Cl}: 0.58$ to 1.46 ) and CV events (RR: $1.01 ; 95 \% \mathrm{Cl}: 0.46$ to 2.22 ).

Conclusion A low-salt diet seems to reduce the risk for renal composite outcome events in patients with CKD. However, no compelling evidence indicated that such a diet would reduce the eGFR decline rate, proteinuria, incidence of all-cause mortality and CV events. Further, more definitive studies are needed.

PROSPERO registration number CRD42017072395.

\section{INTRODUCTION}

Chronic kidney disease (CKD) has become an important global public health burden.

\section{Strengths and limitations of this study}

This is the first meta-analysis of the effect of a lowsalt diet on hard clinical outcomes, such as renal composite outcomes, all-cause mortality and cardiovascular events in patients with chronic kidney disease.

- We included cohort studies and randomised controlled trials without language restriction, and screened reference lists from included articles, maximising the number of studies included in our review.

- The existence of significant heterogeneity may restrict the explanation and clinical application of the results.

- Confounding factors in the observational studies cannot be ignored, which might affect the results of the meta-analysis.

Dietary interventions aimed at delaying the CKD progression and reducing complications play a crucial role in its management. Dietary sodium restriction can augment the antiproteinuric effects of ACE inhibitors (ACEIs)/ angiotensin receptor blockers ${ }^{1}$ and decrease blood pressure (BP). ${ }^{2}$ In the 2021 Kidney disease improving global outcomes (KDIGO) guidelines for glomerulonephritis, reducing dietary sodium intake to $<2 \mathrm{~g}$ /day is a primary tenet for controlling BP and oedema and improving urinary protein excretion independently of medications. However, it is unclear whether these effects translate into a significant total risk decrease in patients with CKD, including kidney failure events, cardiovascular (CV) events and all-cause mortality. In the Chronic Renal Insufficiency Cohort Study, which recruited patients with an estimated glomerular filtration rate (eGFR) $20-70 \mathrm{~mL} /$ min/ $1.73 \mathrm{~m}^{2}$ depending on age, in contrast to the lowest quartile of urinary sodium excretion ( $<116.8 \mathrm{mmol} / 24$ hours $)$, high urinary sodium excretion ( $>194.6 \mathrm{mmol} / 24$ hours $)$ was related to a $54 \%$ risk increase in end-stage renal disease (ESRD) or $50 \%$ decrease in eGFR and with a $43 \%$ risk increase in all-cause mortality. ${ }^{3}$ However, other cohort studies have 
suggested that sodium intake does not affect the progression of CKD to ESRD or eGFR decline ${ }^{45}$ Furthermore, in one study, restricting salt intake to $<6 \mathrm{~g} /$ day was related to an increased risk of all-cause mortality and CV mortality in haemodialysis patients, resulting in an L-shaped association curve. ${ }^{6}$ In the NHANES II Study, which recruited 7154 community elderly people in the USA, restriction of sodium intake to $<2.3 \mathrm{~g}$ daily resulted in increases of $37 \%$ in CV mortality and $28 \%$ in all-cause mortality compared with individuals whose sodium intake was $>2.3 \mathrm{~g}$ daily for more than 13.7 years of follow-up period. ${ }^{7}$ Evidence from randomised controlled trials (RCTs) of the effect of a lowsalt diet in patients with CKD on kidney failure events, CV events and all-cause mortality is controversial. It is hard to make clinical strategy based on results from these studies, considering their small sizes, highly selected patients, short follow-up period and short assessment of hard clinical outcomes. Thus, the effect of dietary salt reduction on clinical endpoints in patients with CKD remains unclear. We performed this systematic review of relevant clinical studies and evaluated the effect of a low-salt diet on renal outcomes, all-cause mortality and CV events in patients with CKD.

\section{MATERIALS AND METHODS}

\section{Data sources and retrieval strategy}

We performed this systematic review following a prespecified protocol registered at the International Prospective Register of Systematic Reviews (online supplemental file $1), 8$ and reporting was performed in accordance with Preferred Reporting Items for Systematic Reviews and Meta-analyses guidelines. ${ }^{9}$ A comprehensive search was conducted using the following databases: MEDLINE by Ovid (1946-July 2021), EMBASE (1966-July 2021) and Cochrane Central Register of Controlled Trials (no date limitation), with related keywords and medical subject headings that included all spellings of "CKD", "RCT", "Cohort Studies", "Sodium Chloride", "Sodium", and "Sodium-Restricted" (see online supplemental file 2 for full search terms). Studies were considered without any language restriction. To ensure an overall literature search, we also screened reference lists from included studies. The ClinicalTrials.gov website was also searched for ongoing, but unpublished studies in this field.

\section{Study screening and outcome evaluation}

We included data from RCTs and cohort studies in which a low-salt diet was given to adults with CKD (as defined by the KDIGO 2012 Clinical Practice Guideline for the Evaluation and Management of CKD) compared with usual therapy or different levels of salt intake. Salt reduction was defined as that recommended by guidelines: sodium $<2.3 \mathrm{~g} /$ day $(<100 \mathrm{mmol}$ sodium, $<6 \mathrm{~g}$ salt $)$, or as defined by the authors of the study.

Predefined outcomes that contained analysable data were as follows: renal composite outcome events, defined as more than $50 \%$ decline in eGFR from baseline during follow-up,,$^{10}$ doubling of serum creatinine or ESRD; the rate of change in eGFR per year; changes in urinary protein or urinary albumin during follow-up, including urinary protein excretion, urinary albumin excretion and urinary albumin/creatinine ratio; all-cause mortality events; CV events, defined as a composite, including fatal or non-fatal myocardial infarction, fatal or non-fatal stroke, coronary artery revascularisation, CV disease and CV death; and changes in systolic blood pressure (SBP) and diastolic blood pressure (DBP).

\section{Data extraction and quality estimation}

Two independent reviewers (HS and XS) extracted data and assessed their quality according to the prespecified protocol. Disagreements were resolved by a third reviewer (LW). Data from all eligible studies were extracted into a spreadsheet. The data sought included characteristics of studies (study type, randomisation method, follow-up time, withdrawals/dropouts), baseline patient traits (age, sex, baseline eGFR and baseline BP), intake of salt and outcome events.

We used Cochrane Collaboration risk-of-bias tool ${ }^{11}$ to assess all potential sources of bias for the included RCTs. Trials were assessed as being at low, some concerns or high risk, and the overall risk of bias generally corresponded to the worst risk of bias in any of the domains. However, if a study was judged to have some concerns about risk of bias for multiple domains, it might be judged as being at high risk for bias overall. We used the Newcastle-Ottawa Scale (NOS) checklist to assess the quality of cohort studies. The NOS contains eight items, categorised into three dimensions: selection of cohorts, comparability of cohorts and assessment of outcome. ${ }^{12}$ A star system is used for semiquantitative assessment of study quality, such that the highest quality studies are awarded a maximum of one star for each item, except the item related to comparability, which can be assigned two stars. The NOS ranges from zero to nine stars.

\section{Data synthesis and analyses}

When dichotomous outcome data from individual trials were analysed, relative risks (RRs) and 95\% CIs were used. If the RR and 95\% CI for an individual study were unavailable in the original article, we calculated them using event numbers extracted from each study before data pooling. In calculating the RR values, we used the total number of patients randomised in each group as the corresponding denominator. Continuous outcome data from individual trials were analysed using mean differences (MDs) with 95\% CIs to pool eGFR, and standardised mean differences (SMDs) with 95\% CIs were used to pool the proteinuria or albuminuria data. When continuous outcome data were analysed, the change in MD between baseline and end of treatment was used. If these data were not available in the studies, we calculated using correlations estimated from other included studies that had a similar follow-up and reported their results in considerable details according to the imputed formulation and its related interpretations in the Cochrane Handbook. ${ }^{13}$ 
Given the poor stability of the DerSimonian-Laird procedure for a small number of studies, ${ }^{14}$ we used the empirical Bayes procedure ${ }^{15}$ to estimate all outcomes. We also used DerSimonian-Laird random-effects mode ${ }^{16}$ and restricted maximum likelihood approach ${ }^{17}$ to assess the summary effects as part of the sensitivity analyses. Considering the inevitable heterogeneity among studies, subgroup, meta-regression and sensitivity analyses were performed. Subgroup analyses were performed according to a prespecified protocol, including study type, baseline eGFR, baseline BP and comparator of control group. In addition, we performed sensitivity analyses excluding studies with a sample size of $<50$, studies with a follow-up of $<12$ months, studies of low quality (high risk for overall bias in RCTs, NOS $<5$ stars for cohort studies) and studies with extreme outliers. Heterogeneity among studies was evaluated using the $\mathrm{I}^{2}$ or $\tau^{2}$ statistic. $\mathrm{I}^{2}$ values of $25 \%$, $50 \%$, and $75 \%$, respectively, represent low, moderate, and high heterogeneity. Publication bias was assessed using a funnel plot, Egger's test or Begg's test, and a p value of $<0.05$ indicated obvious publication bias. Stata V.15.0 (StataCorp, College Station, Texas, USA) was used for statistical analyses, and a two-sided $p$ value of $<0.05$ was considered indicative of significance.

\section{Patient and public involvement}

No patient or member of the public was involved in the development of the research question, selection of the outcome measures, design and implementation of the study or interpretation of the results.

\section{RESULTS}

\section{Overview of included trials}

We identified 9948 potential relevant records. After removing duplicates, we screened the titles and abstracts, and the full text of 230 publications were reviewed. As shown in figure 1, online supplemental files 3 and 4 , a total of 33 eligible studies ${ }^{3-6}{ }^{18-48}$ including 13 RCTs ${ }^{182} 2025-27313235-4045$ and 19 cohort studies ${ }^{3-6} 19$ 21-24 28303334 41-44 46-48 reported in 35 publications with 101077 participants were included in our review. The median follow-up was 6 months (IQR 3-21 months). Participants were enrolled at an average age of 56 years, and male participants accounted for $60 \%$ of the total. The average eGFR of the participants was $47.45 \mathrm{~mL} / \mathrm{min} / 1.73 \mathrm{~m}^{2}$. Twelve studies were usual care or usual diet controlled, and 21 compared different levels of sodium intake.

The risk of bias varied substantially across the RCTs. The results from the Cochrane Collaboration risk-of-bias tool are shown in online supplemental file 5. Bias arising from the randomisation process was considered to be low in $61.54 \%$ of the RCTs, and all studies had a low risk of bias due to missing outcome data, bias in measurement of the outcome and bias in selection of the reported results. In terms of overall bias, $61.54 \%$ of the trials were considered to have low risk, and $38.46 \%$ had some concern of bias.

According to the NOS checklist, all included cohort studies were generally high quality and awarded six to eight stars (online supplemental file 6).

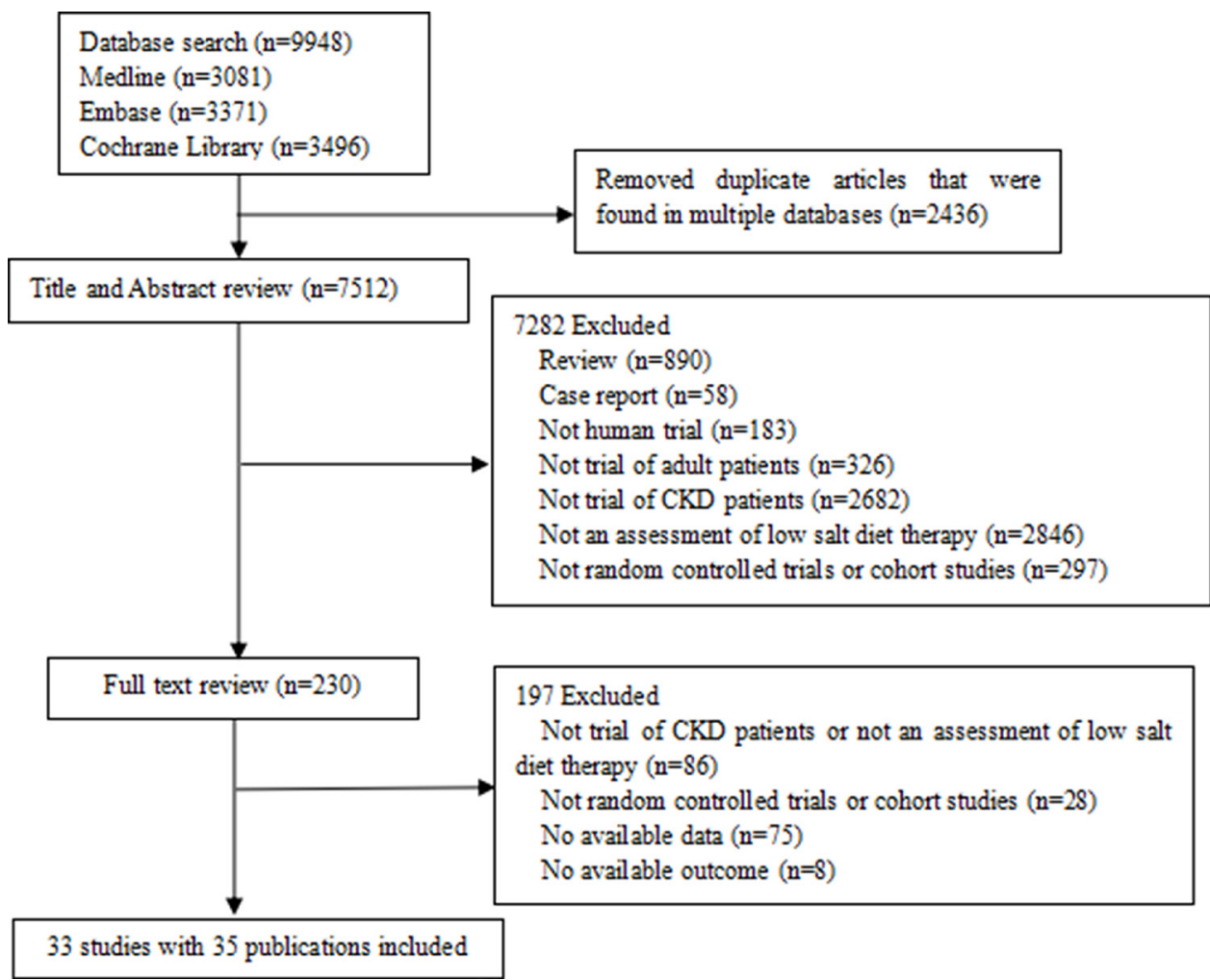

Figure 1 PRISMA flow chart of the included studies. CKD, chronic kidney disease; PRISMA, Preferred Reporting Items for Systematic Reviews and Meta-analyses. 


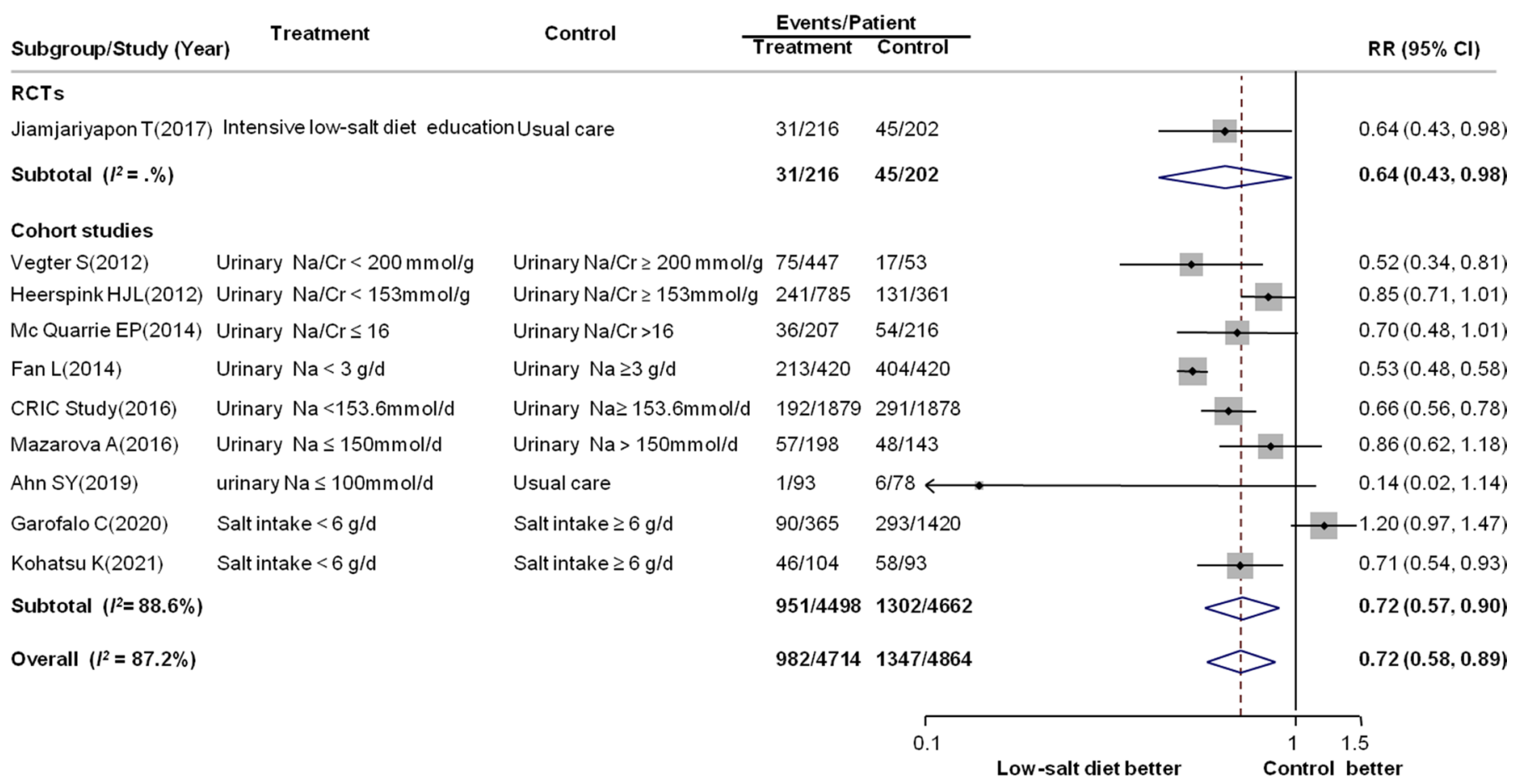

Figure 2 Forest plot for renal composite outcome events. Renal composite outcome events were defined as more than 50\% decline in eGFR from baseline during follow-up or a doubling of serum levels of $\mathrm{Cr}$ or ESRD. Cr, creatinine; eGFR, estimated glomerular filtration rate; ESRD, end-stage renal disease; Na, sodium; RCTs, randomised controlled trials; RR, relative risk.

\section{Effect of a low-salt diet on renal outcomes}

One RCT and nine observational studies including 9578 patients with 2329 events compared a low-salt diet with controls in terms of preventing renal composite outcome events. As shown in figure 2, compared with controls, a low-salt diet produced a $28 \%$ reduction in the risk for renal composite outcome events (RR: $0.72 ; 95 \%$ CI: 0.58 to $0.89)$, with substantial heterogeneity $\left(\mathrm{I}^{2}=87.2 \%, \mathrm{p}<0.001\right)$. Subgroup analyses and meta-regression were conducted to assess the source of heterogeneity. No significant difference between RCTs and observational studies was found, whereas the effect sizes were greater in studies that enrolled patients aged $<58$ years $(\mathrm{p}=0.03)$ (table 1$)$. In univariate meta-regression, no clear relationship between 24-hour sodium in urine $(\mathrm{p}=0.80)$ or baseline eGFR $(\mathrm{p}=0.51)$ and the reduction in renal composite outcome events was observed (online supplemental files 7 and 8).

Five RCTs and seven observational studies with a total of 3287 individuals reported data regarding the effect of a lowsalt diet on the rate of change in eGFR. The diet did not have a beneficial effect on the rate of change in eGFR (MD: 1.16; 95\% CI: -2.02 to 4.33$)$. There was evidence of significant heterogeneity across the included studies $\left(\mathrm{I}^{2}=98.0 \%\right.$, $\mathrm{p}<0.001$ ) (figure 3). Subgroup analyses showed that there was no statistical heterogeneity according to prespecified characteristics (online supplemental file 9).

Data on the effect of a low-salt diet on proteinuria or albuminuria were available in 13 studies, with 2922 participants. The SMD for change in proteinuria or albuminuria was not statistically significant at -0.71 (95\% CI: -1.66 to 0.24 ) compared with controls, with significant heterogeneity
$\left(\mathrm{I}^{2}=97.3 \%, \mathrm{p}=0.009\right)$ (figure 4$)$. No statistical heterogeneity was found in subgroup analyses of the effect of a low-salt diet on proteinuria or albuminuria (online supplemental file 9).

\section{Effect of a low-salt diet on all-cause mortality and CV events}

Data on the effect of low-salt diet on all-cause mortality and CV events were available for nine studies (two RCTs and seven observational studies) and five studies (one RCT and four observational studies), respectively. There was no effect of a low-salt diet on the risk of all-cause mortality (RR: 0.92; 95\% CI: 0.53 to 1.46 ; 95997 participants and 4455 events) or CV events (RR: 1.01 ; $95 \%$ CI: 0.46 to 2.22; 93573 participants and 1989 events) compared with controls, with significant heterogeneity across the included studies $\left(\mathrm{I}^{2}=98.0 \%\right.$, $97.6 \%$ for all-cause mortality and CV events, respectively, both p values for heterogeneity <0.001) (figure 5). A greater benefit was found in studies that included patients with an eGFR of $30-59 \mathrm{~mL} / \mathrm{min} / 1.73 \mathrm{~m}^{2}$ ( $\mathrm{p}$ for subgroup heterogeneity $=0.02$ ) in the subgroup analyses of $\mathrm{CV}$ events (online supplemental file 9 ).

\section{Effect of a low-salt diet on BP}

Twenty studies reported the effect of a low-salt diet on BP, including 19 studies (3608 participants) for SBP and 18 studies (3608 participants) for DBP. The MD changes in SBP and DBP were statistically significant at $-5.81 \mathrm{~mm} \mathrm{Hg}$ (95\% CI: -9.63 to -1.99$)$ and $-2.3 \mathrm{~mm} \mathrm{Hg}(95 \% \mathrm{CI},-3.61$ to -0.98 ), respectively (online supplemental files 10 and 11). Significant heterogeneity across the included studies was found $\left(\mathrm{I}^{2}=87.1 \%\right.$ and $68.2 \%$ for $\mathrm{SBP}$ and $\mathrm{DBP}$, respectively; both $\mathrm{p}$ values for heterogeneity $<0.05)$. Subgroup 
Table 1 Results of subgroup analysis of renal composite outcome events

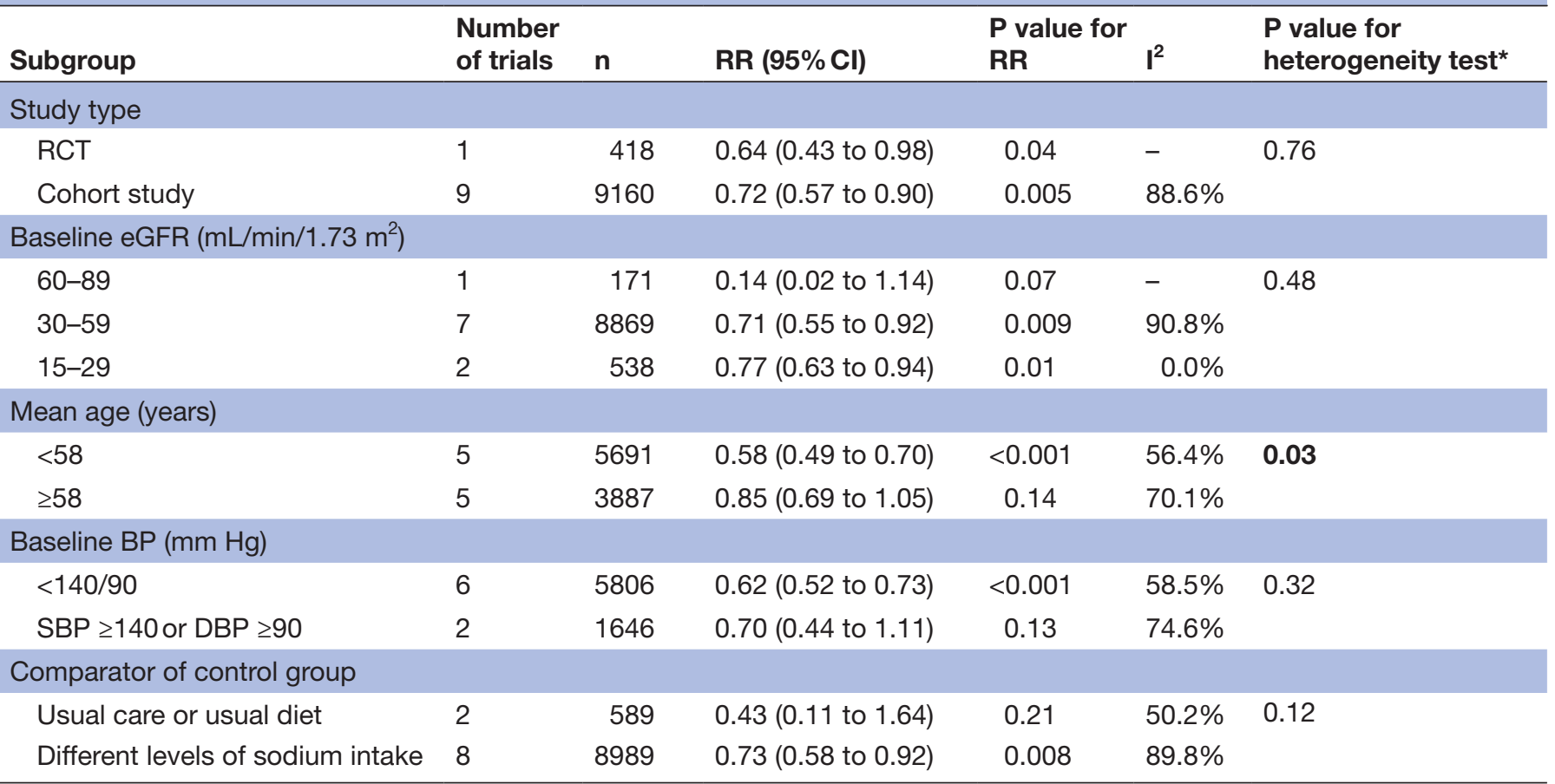

The bold $\mathrm{P}$ value indicates that there are statistical differences between subgroups.

${ }^{*} \mathrm{P}$ value calculated by meta-regression.

BP, blood pressure; DBP, diastolic blood pressure; eGFR, estimated glomerular filtration rate; $n$, number of patients; $R C T$, randomised controlled trial; RR, relative risk; SBP, systolic blood pressure.

analyses showed that the association between the change in SBP or DBP and a low-salt diet was modified by the baseline BP. Patients with a baseline SBP $>140 \mathrm{~mm} \mathrm{Hg}$ or DBP $>90 \mathrm{~mm} \mathrm{Hg}$ had an average reduction in SBP and
DBP of 10 and $5 \mathrm{~mm} \mathrm{Hg}$, respectively (online supplemental file 9).

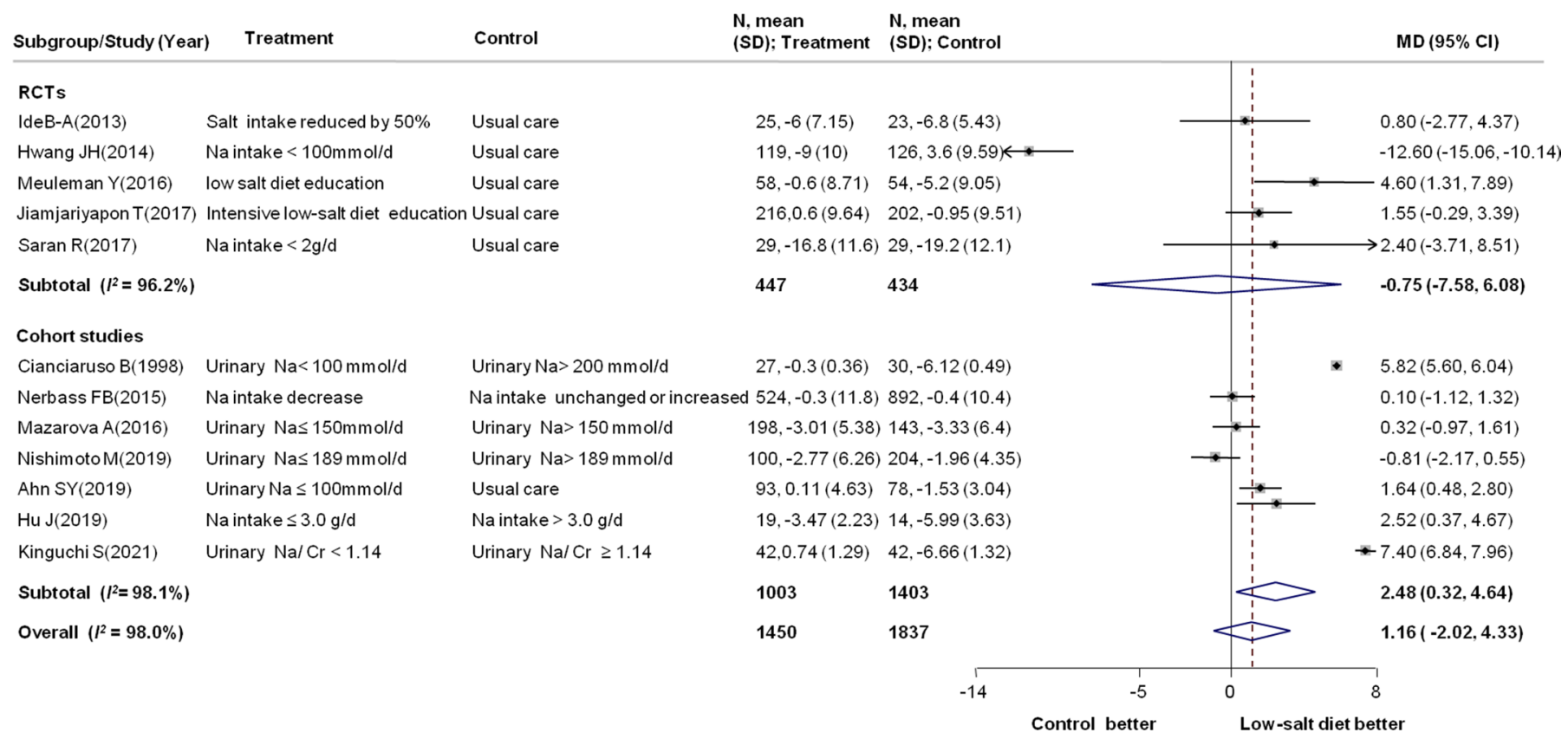

Figure 3 Forest plot of the rate of change in estimated glomerular filtration rate. Cr, creatinine; MD, mean difference; Na, sodium; RCTs, randomised controlled trials. 


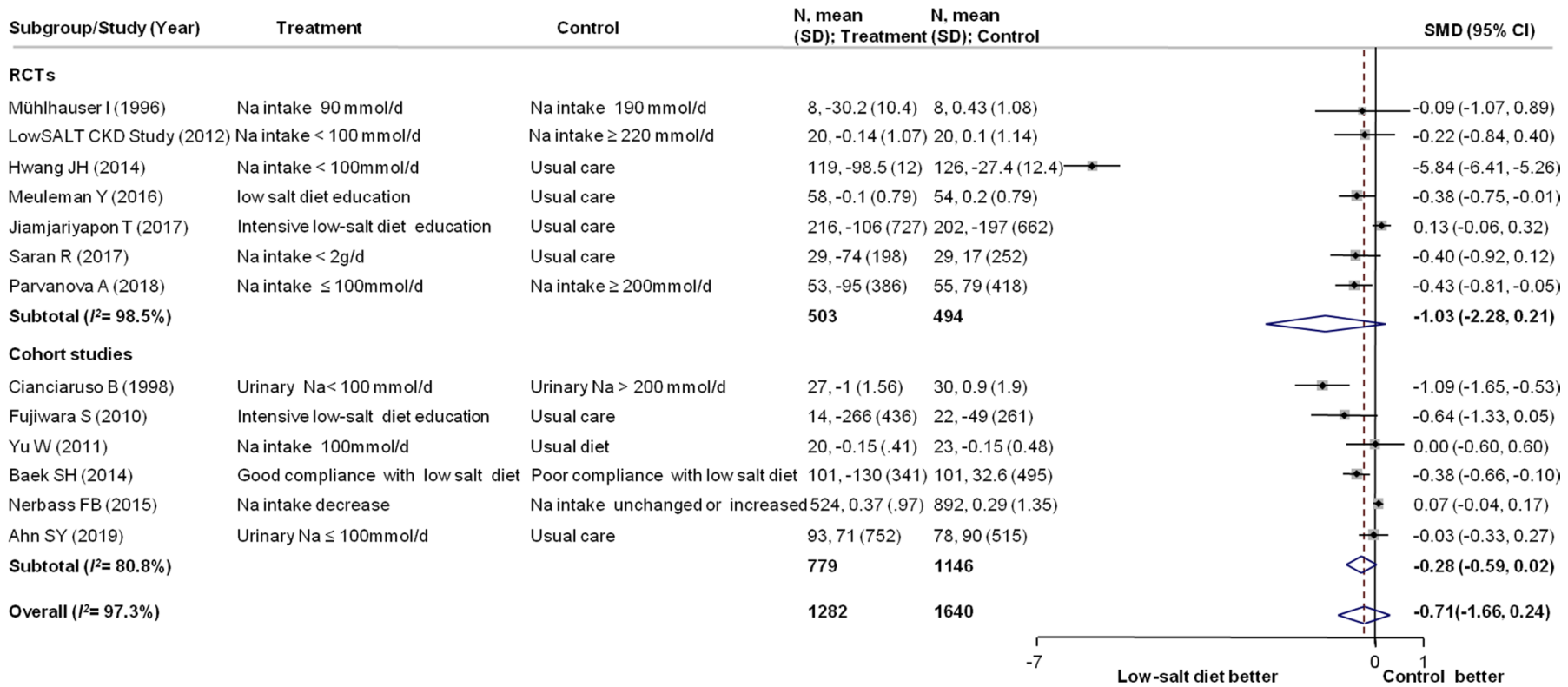

Figure 4 Forest plot for the change in proteinuria or albuminuria. CKD, chronic kidney disease; Na, sodium; RCTs, randomised controlled trials; SMD, standard mean difference.

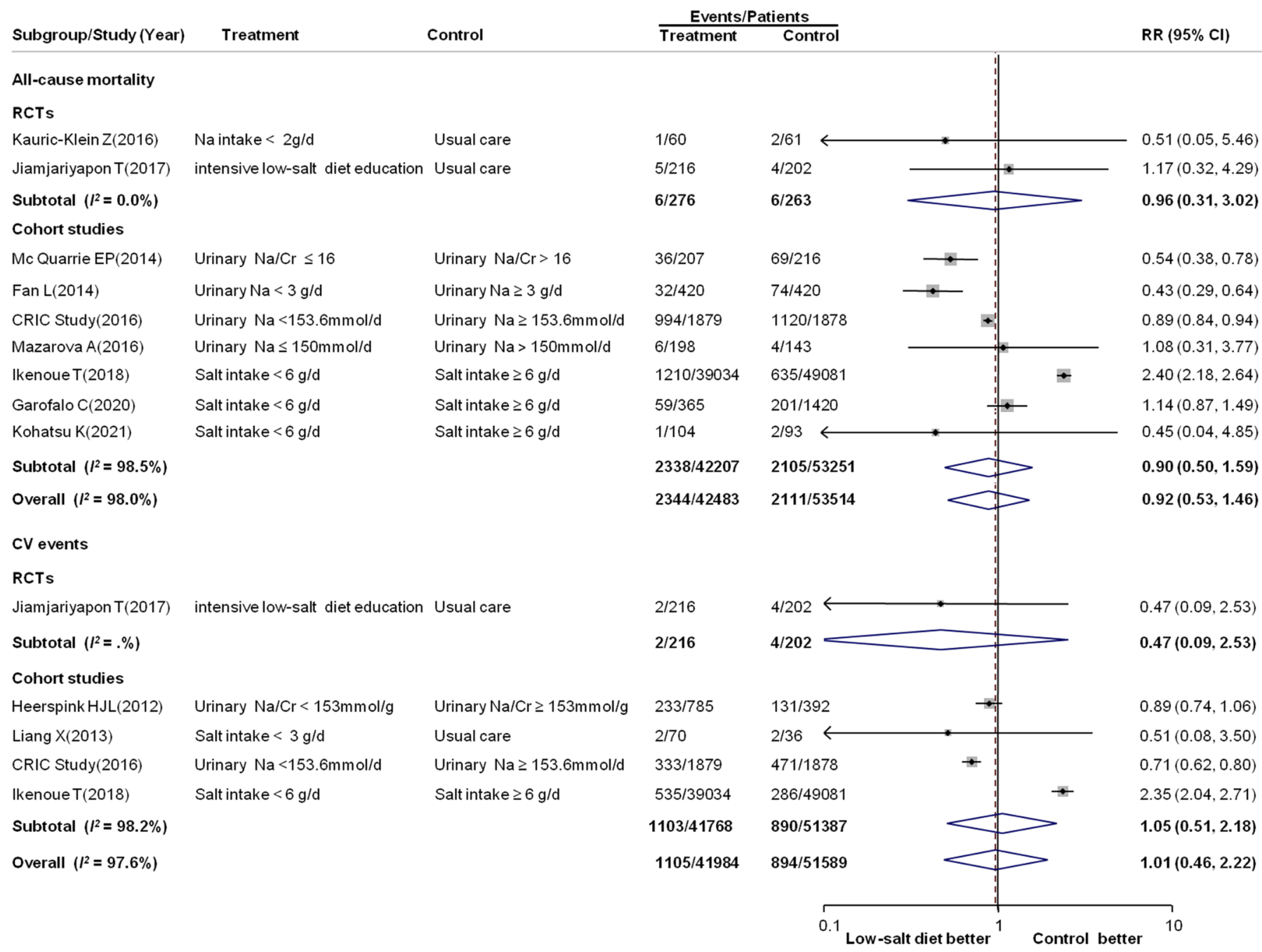

Figure 5 Forest plot for all-cause mortality and cardiovascular (CV) events. CV events were defined as a composite of fatal or non-fatal myocardial infarction, fatal or non-fatal stroke, coronary artery revascularisation, CV disease and CV death. $\mathrm{Cr}$, creatinine; $\mathrm{Na}$, sodium; RCTs, randomised controlled trials; RR, relative risk. 


\section{Sensitivity analyses and publication bias}

The results of all sensitivity analyses were robust (online supplemental file 12). The most notable exception was that the effect of a low-salt diet on the rate of change in eGFR became significant when studies with extreme outliers were omitted, and proteinuria or albuminuria became significant when the DerSimonian-Laird method was used to pool data. The risk of publication bias was significant for the rate of change in eGFR, proteinuria or albuminuria, all-cause mortality, CV events and BP (online supplemental files 13 and 14).

\section{DISCUSSION}

Behavioural modifications can delay CKD progression to ESRD, and dietary salt restriction is one of the main concerns. Our meta-analysis showed that compared with the control groups, a low-salt diet reduced renal composite outcome events and lowered BP (SBP and DBP) in patients with CKD. No significant effects were observed on the eGFR decline rate, change in proteinuria, risk of all-cause mortality and CV events. The results were concordant across major subgroups and sensitivity analyses. However, the significant heterogeneity among the studies may restrict their explanation and clinical practice.

A prior review of 16 studies on salt consumption and kidney disease investigated whether variation in dietary sodium intake affects kidney outcomes in patients with CKD. ${ }^{49}$ Increased salt consumption was related to increased albuminuria and the possibility of a decrease in GFR. In $2015^{50}$ and $2018,{ }^{51}$ two meta-analyses of RCTs suggested that moderate dietary salt limitation obviously reduced $\mathrm{BP}$ and proteinuria. However, it is unclear whether these benefits convert to clinically obvious reductions in renal composite outcome events, all-cause mortality events and CV events. Our meta-analysis of 33 studies (13 RCTs and 20 observational studies), with more than 90000 patients and 2329 renal composite outcome events, showed that compared with the control groups, a low-salt diet produced a significant $28 \%$ reduction in renal composite outcome events despite marked heterogeneity. Furthermore, the significant effect of a low-salt diet on BP (decreases in SBP and DBP of 5.8 and $2.3 \mathrm{~mm}$ $\mathrm{Hg}$, respectively) was confirmed and is an important target for slowing the progression of CKD to ESRD. The benefit of proteinuria from low-salt diet became nonsignificant when the restricted maximum likelihood or empirical Bayes method was used to pool data. The standard DerSimonian-Laird procedure, which was applied in previous meta-analyses, can be unstable with small numbers of studies. ${ }^{1452}$

Statistical heterogeneity was found in all outcomes, and clinical heterogeneity was an inevitable issue. Lowsalt diets are not palatable for some patients. ${ }^{53}$ Therefore, it is hard to confirm persistent compliance with a salt restriction during long-term follow-up time. Sodium intake was quantified using different methods (such as, 24-hour urine collection, spot urine collection or dietary questionnaire). Because diuretics influence urine sodium excretion and eGFR, it would influence the accuracy of quantified salt intake. Ten studies had data on diuretic use, but included only the percentages of patients using diuretics in the intervention and control groups. The percentage of patients using diuretics was similar in most of the studies. There were no data on the type and dose of diuretics, or their associations with the eGFR. These inherent problems were reflected in paradoxically opposite findings, especially of observational studies with several confounding factors. Only three studies (one RCT and two observational studies) simultaneously reported the outcomes of eGFR and renal composite outcome events. Furthermore, the different follow-up periods $(52$ months of renal composite outcome events and 12 months of eGFR) should be noted. The inconsistent results of eGFR and renal composite outcome events may be explained in part by the heterogeneity, which likely limited the interpretation and generalisability of our results.

In patients with CKD, BP is typically sodium sensitive, and renin-angiotensin-aldosterone system (RAAS) blockers are considered the first-line therapy for hypertension and proteinuria. The function of RAAS blockers is weakened by high salt intake ${ }^{54-56}$; therefore, the significant effect of dietary sodium restriction on lowering BP and supporting the function of RAAS blockers was speculated to play a very important part in reducing the risk of renal failure. Interestingly, a BP-independent effect of dietary sodium on the kidney is substantial by data in healthy volunteers, in which dietary sodium restriction reduces albuminuria to within the normal range, without a detectable effect on $\mathrm{BP}^{57}$ Similarly, proteinuria reduction by sodium restriction remained significant after adjustment for the decrease in $\mathrm{BP} .{ }^{56}$ These results suggest an independent renoprotective function of a low-salt diet.

There was no convincing evidence that a low-salt diet was related to a lower incidence of all-cause mortality and $\mathrm{CV}$ events. Limited data on all-cause mortality (nine studies with 4455 events) and CV events (five studies with 1989 events) in the meta-analysis might introduce a risk of false-negative results because of low statistical power. A greater benefit for CV events was found in studies that included patients with an eGFR $30-59 \mathrm{~mL} / \mathrm{min} / 1.73 \mathrm{~m}^{2}$ in subgroup analyses, whereas the effects were not significant in patients with an eGFR $<15 \mathrm{~mL} / \mathrm{min} / 1.73 \mathrm{~m}^{2}$. The substantial subgroup heterogeneity $(\mathrm{p}=0.02)$ could be attributable to more CV risk factors in patients with ESRD than in those with an eGFR $30-59 \mathrm{~mL} / \mathrm{min} / 1.73 \mathrm{~m}^{2}$. The benefit of a low-salt diet for CV events did not outweigh the increase in $\mathrm{CV}$ events caused by more risk factors (such as hyperuricemia, hyperphosphatemia and vascular calcification). Therefore, education on the importance of a low-salt diet should be emphasised in patients with early-stage CKD.

Observational data showing a J-curve between sodium intake and renal and CV outcomes have raised concern regarding the safety of rigorous sodium restriction. ${ }^{58-60}$ 
It is uncertain whether the presence of CKD modifies this association. In our meta-regression analysis, no clear relationship between 24-hour sodium in urine $(\mathrm{p}=0.80)$ or baseline eGFR $(\mathrm{p}=0.51)$ and the reduction in renal composite outcome events was observed. A combination of angiotensin-converting enzymes (ACEIs) and strict sodium control decreased BP, proteinuria and glomerular lesion but exacerbated tubule-interstitial injury in experimental renal disease. ${ }^{61}$ This could explain the reason of worse renal outcomes by very low salt intake. High or extremely low-dietary salt intake might be inappropriate for patients with CKD. KDIGO recommends a daily sodium intake of $<2.0 \mathrm{~g} /$ day in patients with CKD without a clear lower limit. Therefore, an appropriate dietary salt intake should be discussed with patients with CKD. Further studies are necessary to identify the optimal dietary salt intake for renoprotection in patients with CKD.

This study had several potential limitations. First, the existence of statistical and clinical heterogeneity may raise concerns regarding validity, although we attempted to resolve these concerns by performing subgroup and sensitivity analyses. Heterogeneity in study populations hampers meaningful interpretation of the results. Because the CKD stages varied markedly, it is difficult to stratify the outcome analysis by CKD stage. Instead, we performed subgroup analyses according to baseline eGFR. Second, unlike RCTs, cohort studies are prone to selection bias. Therefore, pooling the results from RCTs and cohort studies will bias the results of the meta-analysis. We performed a subgroup analysis according to study design. There was no statistical heterogeneity in the outcomes according to study design. However, a low-salt diet did not exert a beneficial effect on the rate of change in eGFR in RCTs (MD: -0.75 ; $95 \%$ CI: -7.58 to 6.08 ) but did in eGFR in cohort studies (MD: 2.48; 95\% CI: 0.32 to 4.64 ). Third, many of the included low-quality studies had small sample sizes and low incidences of events. Consequently, the uncertainty in the analyses was increased, resulting in wide CIs of effect measures. Fourth, confounding factors in the observational studies cannot be neglected, because they might affect the results. Therefore, the findings of this study should be viewed as hypothesis generating and need to be confirmed by further studies.

\section{CONCLUSION}

This meta-analysis suggests that a low-salt diet may reduce the risk of renal composite outcome events in patients with CKD. However, there was no compelling evidence that a low-salt diet reduces the rate of eGFR decline, proteinuria, incidence of all-cause mortality and CV events. The optimal dietary salt intake for patients with different CKD stages is unclear. Further well-designed RCTs targeting patients with different CKDs are required.

Acknowledgements We thank Textcheck for proofreading and editing the English text of the manuscript.
Contributors All authors take responsibility for the integrity of the data and the accuracy of data analysis. Study concept and design-LW, XS, HS and CL. Extraction, analysis and interpretation of data-HS and XS. Drafting of the manuscript-HS and LW. Critical revision of the manuscript-LW and XS. Statistical analysis- HS, CL and WG. Technical support-LW, XS and WG. All authors have read and agreed to the submission of this journal. All authors had full access to all the data in the study and had final responsibility for the decision to submit for publication.

Funding Support was provided by the National Natural Science Foundation of China (no. 82000655) and the Science and Technology Project of Shanxi Province (no. 201801D121218).

Disclaimer The funders had no role in the decision to publish or in preparation of the manuscript.

Competing interests None declared.

Patient consent for publication Not required.

Ethics approval This study does not involve human participants.

Provenance and peer review Not commissioned; externally peer reviewed.

Data availability statement All data relevant to the study are included in the article or uploaded as supplemental information. The data used to support the findings of this study are included within the article and supplemental information.

Supplemental material This content has been supplied by the author(s). It has not been vetted by BMJ Publishing Group Limited (BMJ) and may not have been peer-reviewed. Any opinions or recommendations discussed are solely those of the author(s) and are not endorsed by BMJ. BMJ disclaims all liability and responsibility arising from any reliance placed on the content. Where the content includes any translated material, BMJ does not warrant the accuracy and reliability of the translations (including but not limited to local regulations, clinical guidelines, terminology, drug names and drug dosages), and is not responsible for any error and/or omissions arising from translation and adaptation or otherwise.

Open access This is an open access article distributed in accordance with the Creative Commons Attribution Non Commercial (CC BY-NC 4.0) license, which permits others to distribute, remix, adapt, build upon this work non-commercially, and license their derivative works on different terms, provided the original work is properly cited, appropriate credit is given, any changes made indicated, and the use is non-commercial. See: http://creativecommons.org/licenses/by-nc/4.0/.

ORCID iD

Honghong Shi http://orcid.org/0000-0003-1385-5270

\section{REFERENCES}

1 Heeg JE, de Jong PE, van der Hem GK, et al. Efficacy and variability of the antiproteinuric effect of ACE inhibition by lisinopril. Kidney Int 1989;36:272-9.

2 Sacks FM, Svetkey LP, Vollmer WM, et al. Effects on blood pressure of reduced dietary sodium and the dietary approaches to stop hypertension (DASH) diet. DASH-Sodium Collaborative Research Group. N Engl J Med 2001;344:3-10.

$3 \mathrm{He} \mathrm{J}$, Mills KT, Appel LJ, et al. Urinary sodium and potassium excretion and CKD progression. J Am Soc Nephrol 2016;27:1202-12.

4 Fan L, Tighiouart H, Levey AS, et al. Urinary sodium excretion and kidney failure in nondiabetic chronic kidney disease. Kidney Int 2014;86:582-8.

5 Mazarova A, Molnar AO, Akbari A, et al. The association of urinary sodium excretion and the need for renal replacement therapy in advanced chronic kidney disease: a cohort study. BMC Nephrol 2016;17:123.

6 Ikenoue T, Koike K, Fukuma S, et al. Salt intake and all-cause mortality in hemodialysis patients. Am J Nephrol 2018;48:87-95.

7 Cohen HW, Hailpern SM, Fang J, et al. Sodium intake and mortality in the NHANES II follow-up study. Am J Med 2006;119:275.e7-14.

8 et alLi C, Wang L, Su X. Effect of dietary sodium restriction on renal outcome in patients with chronic kidney disease. PROSPERO 2017 CRD42017072395. Available: http://www.crd.york.ac.uk/ PROSPERO/display_record.php?ID=CRD42017072395

9 Moher D, Liberati A, Tetzlaff J, et al. Preferred reporting items for systematic reviews and meta-analyses: the PRISMA statement. PLoS Med 2009;6:e1000097.

10 Coresh J, Turin TC, Matsushita K, et al. Decline in estimated glomerular filtration rate and subsequent risk of end-stage renal disease and mortality. JAMA 2014;311:2518-31. 
11 Sterne JAC, Savović J, Page MJ, et al. RoB 2: a revised tool for assessing risk of bias in randomised trials. BMJ 2019;366:I4898.

12 Stang A. Critical evaluation of the Newcastle-Ottawa scale for the assessment of the quality of nonrandomized studies in metaanalyses. Eur J Epidemiol 2010;25:603-5.

13 Higgins JP, Green S. Cochrane Handbook for systematic reviews of interventions. version 5.1.0. The Cochrane Collaboration, 2011.

14 Cornell JE, Mulrow CD, Localio R, et al. Random-effects metaanalysis of inconsistent effects: a time for change. Ann Intern Med 2014;160:267-70.

15 Friston KJ, Penny W, Phillips C, et al. Classical and Bayesian inference in neuroimaging: theory. Neuroimage 2002;16:465-83.

16 DerSimonian R, Laird N. Meta-analysis in clinical trials revisited. Contemp Clin Trials 2015;45:139-45.

17 Harville DA. Maximum likelihood approaches to variance component estimation and to related problems. J Am Stat Assoc 1977;72:320-38.

18 Mühlhauser I, Prange K, Sawicki PT, et al. Effects of dietary sodium on blood pressure in IDDM patients with nephropathy. Diabetologia 1996;39:212-9.

19 Cianciaruso B, Bellizzi V, Minutolo R, et al. Salt intake and renal outcome in patients with progressive renal disease. Miner Electrolyte Metab 1998;24:296-301.

20 Keven K, Yalçin S, Canbakan B, et al. The impact of daily sodium intake on posttransplant hypertension in kidney allograft recipients. Transplant Proc 2006;38:1323-6.

21 Fujiwara S, Kotani K, Brantley PJ, et al. Dietary salt reduction in rural patients with albuminurea using family and community support: the Mima study. Asia Pac Fam Med 2010;9:6.

22 Yu W, Luying S, Haiyan W, et al. Importance and benefits of dietary sodium restriction in the management of chronic kidney disease patients: experience from a single Chinese center. Int Urol Nephrol 2012;44:549-56.

23 Vegter S, Perna A, Postma MJ, et al. Sodium intake, ACE inhibition, and progression to ESRD. J Am Soc Nephrol 2012;23:165-73.

24 Lambers Heerspink HJ, Holtkamp FA, Parving $\mathrm{H}-\mathrm{H}$, et al. Moderation of dietary sodium potentiates the renal and cardiovascular protective effects of angiotensin receptor blockers. Kidney Int 2012;82:330-7.

25 McMahon EJ, Bauer JD, Hawley CM, et al. A randomized trial of dietary sodium restriction in CKD. J Am Soc Nephrol 2013;24:2096-103.

26 Campbell KL, Johnson DW, Bauer JD, et al. A randomized trial of sodium-restriction on kidney function, fluid volume and adipokines in CKD patients. BMC Nephrol 2014;15:57.

27 de Brito-Ashurst I, Perry L, Sanders TAB, et al. The role of salt intake and salt sensitivity in the management of hypertension in South Asian people with chronic kidney disease: a randomised controlled trial. Heart 2013;99:1256-60.

28 Liang X, Wang W, Li H. Water and sodium restriction on cardiovascular disease in young chronic hemodialysis patients. Chin Med J 2013;126:1667-72.

29 McQuarrie EP, Traynor JP, Taylor AH, et al. Association between urinary sodium, creatinine, albumin, and long-term survival in chronic kidney disease. Hypertension 2014;64:111-7.

30 Baek SH, Kim S, Kim DK, et al. A low-salt diet increases the estimated net endogenous acid production in nondiabetic chronic kidney disease patients treated with angiotensin receptor blockade. Nephron Clin Pract 2014;128:407-13.

31 Hwang JH, Chin HJ, Kim S, et al. Effects of intensive low-salt diet education on albuminuria among nondiabetic patients with hypertension treated with olmesartan: a single-blinded randomized, controlled trial. Clin J Am Soc Nephrol 2014;9:2059-69.

32 Rodrigues Telini LS, de Carvalho Beduschi G, Caramori JCT, et al. Effect of dietary sodium restriction on body water, blood pressure, and inflammation in hemodialysis patients: a prospective randomized controlled study. Int Urol Nephrol 2014;46:91-7.

33 Nerbass FB, Pecoits-Filho R, Mclntyre NJ, et al. Reduction in sodium intake is independently associated with improved blood pressure control in people with chronic kidney disease in primary care. $\mathrm{Br} J$ Nutr 2015;114:936-42.

34 Mills KT, Chen J, Yang W, et al. Sodium excretion and the risk of cardiovascular disease in patients with chronic kidney disease. JAMA 2016;315:2200-10.

35 Kauric-Klein Z, Peters RM, Yarandi HN. Self-Efficacy and blood pressure self-care behaviors in patients on chronic hemodialysis. West J Nurs Res 2017;39:886-905.

36 Meuleman Y, Hoekstra T, Dekker FW, et al. Sodium restriction in patients with CKD: a randomized controlled trial of self-management support. Am J Kidney Dis 2017;69:576-86.

37 Jiamjariyapon $\mathrm{T}$, Ingsathit A, Pongpirul K, et al. Effectiveness of integrated care on delaying progression of stage 3-4 chronic kidney disease in rural communities of Thailand (escort study): a cluster randomized controlled trial. BMC Nephrol 2017;18:83.

38 Saran R, Padilla RL, Gillespie BW, et al. A randomized crossover trial of dietary sodium restriction in stage 3-4 CKD. Clin J Am Soc Nephrol 2017;12:399-407.

39 Huang B, Li Z, Wang Y, et al. Effectiveness of self-management support in maintenance haemodialysis patients with hypertension: a pilot cluster randomized controlled trial. Nephrology 2018;23:755-63.

40 Parvanova A, Trillini M, Podestà MA, et al. Moderate salt restriction with or without paricalcitol in type 2 diabetes and losartan-resistant macroalbuminuria (proceed): a randomised, double-blind, placebocontrolled, crossover trial. Lancet Diabetes Endocrinol 2018;6:27-40.

41 Martinez MG, Dos Santos Silva V, do Valle AP, et al. Association between sodium intake and urinary fractional albumin and immunoglobulin $\mathrm{G}$ excretion in chronic Nondialytic renal disease: a prospective longitudinal study. Nephron 2019;143:62-7.

42 Nishimoto M, Ohtsu H, Marumo T, et al. Mineralocorticoid receptor blockade suppresses dietary salt-induced ACEI/ARB-resistant albuminuria in non-diabetic hypertension: a sub-analysis of evaluate study. Hypertens Res 2019;42:514-21.

43 Ahn SY, Kim DK, Park JH, et al. Long-Term effects of intensive low-salt diet education on deterioration of glomerular filtration rate among non-diabetic hypertensive patients with chronic kidney disease. Kidney Blood Press Res 2019;44:1101-14.

$44 \mathrm{Hu} \mathrm{J}, \mathrm{Hu} \mathrm{L}$, Gong N, et al. [Effect of dietary sodium intake on residual renal function in patients undergoing peritoneal dialysis: a prospective study of 33 cases]. Nan Fang Yi Ke Da Xue Xue Bao 2019;39:657-64.

45 Humalda JK, Klaassen G, de Vries H, et al. A self-management approach for dietary sodium restriction in patients with CKD: a randomized controlled trial. Am J Kidney Dis 2020;75:847-56.

46 Garofalo C, Provenzano M, Andreucci M, et al. Predictive effect of salt intake on patient and kidney survival in non-dialysis CKD: competing risk analysis in older versus younger patients under nephrology care. Nephrol Dial Transplant 2021;36:2232-40.

47 Kinguchi S, Wakui H, Ito $\mathrm{Y}$, et al. Relationship between basal sodium intake and the effects of dapagliflozin in albuminuric diabetic kidney disease. Sci Rep 2021;11:951

48 Kohatsu K, Shimizu S, Shibagaki Y, et al. Association between daily urinary sodium excretion, ratio of extracellular Water-to-Total body water ratio, and kidney outcome in patients with chronic kidney disease. Nutrients 2021;13:650.

49 Jones-Burton C, Mishra SI, Fink JC, et al. An in-depth review of the evidence linking dietary salt intake and progression of chronic kidney disease. Am J Nephrol 2006;26:268-75.

50 McMahon EJ, Campbell KL, Bauer JD, et al. Altered dietary salt intake for people with chronic kidney disease. Cochrane Database Syst Rev 2015:CD010070.

51 Garofalo C, Borrelli S, Provenzano M, et al. Dietary salt restriction in chronic kidney disease: a meta-analysis of randomized clinical trials. Nutrients 2018;10:732.

52 Veroniki AA, Jackson D, Viechtbauer W, et al. Methods to estimate the between-study variance and its uncertainty in meta-analysis. Res Synth Methods 2016;7:55-79.

53 Welch JL, Bennett SJ, Delp RL, et al. Benefits of and barriers to dietary sodium adherence. West $J$ Nurs Res 2006;28:162-80.

54 Navis G, de Jong PE, Donker AJ, et al. Moderate sodium restriction in hypertensive subjects: renal effects of ACE-inhibition. Kidney Int 1987;31:815-9.

55 Vogt L, Waanders F, Boomsma F, et al. Effects of dietary sodium and hydrochlorothiazide on the antiproteinuric efficacy of losartan. J Am Soc Nephrol 2008;19:999-1007.

56 Slagman MCJ, Waanders F, Hemmelder MH, et al. Moderate dietary sodium restriction added to angiotensin converting enzyme inhibition compared with dual blockade in lowering proteinuria and blood pressure: randomised controlled trial. BMJ 2011;343:d4366.

57 Krikken JA, Lely AT, Bakker SJL, et al. The effect of a shift in sodium intake on renal hemodynamics is determined by body mass index in healthy young men. Kidney Int 2007;71:260-5.

58 O'Donnell M, Mente A, Rangarajan S, et al. Urinary sodium and potassium excretion, mortality, and cardiovascular events. $N$ Engl J Med 2014;371:612-23.

59 Thomas MC, Moran J, Forsblom C, et al. The association between dietary sodium intake, ESRD, and all-cause mortality in patients with type 1 diabetes. Diabetes Care 2011;34:861-6.

60 Ekinci El, Clarke S, Thomas MC, et al. Dietary salt intake and mortality in patients with type 2 diabetes. Diabetes Care 2011;34:703-9.

61 Hamming I, Navis G, Kocks MJA, et al. ACE inhibition has adverse renal effects during dietary sodium restriction in proteinuric and healthy rats. J Pathol 2006;209:129-39. 